\title{
GARDNER-DIAMOND SYNDROME: A CASE REPORT
}

Luiz Felipe Dipe Prates Miranda ${ }^{1, *}$, Rafaela Vieiralves João Izzo Pinto ${ }^{1}$, Débora Rocha de Moura Rodrigues de Aguiar ${ }^{1}$

1.Hospital Federal de Bonsucesso, Rio de Janeiro (RJ), Brazil.

*Corresponding author: luizfelipedipe@gmail.com

\section{BACKGROUND}

Autoerythrocyte sensitization syndrome, also known as Gardner-Diamond syndrome (GDS) is a rare disorder characterized by atraumatic painful ecchymoses. Mostly affects women undergoing stressful situations, such as anxiety and depressive disorders and that's why it is also known as psychogenic purpura.

\section{CASE REPORT}

A 46-year-old female patient with high blood pressure presented otorrhagia and recurrent epistaxis, as well as eyelid ecchymosis and bilateral edema (Fig. 1). She also presented nodular, painful cutaneous lesions in the upper and lower limbs and chest (Fig. 2), which progressed to edematous and erythematous plaques and later to ecchymosis, with complete disappearance in about two weeks. During hospitalization, high anxiety and depression were diagnosed after psychiatric assessment. After an extensive investigation, there wasn't any coagulation disorder identified. Biopsies of the lesion in the right leg were performed with septal panniculitis with neutrophils. Special staining by PAS techniques, silver impregnation and FITE did not show microorganisms or deposits of congophilic material. Diagnostic test for GDS was performed with four intradermal injections in the lumbar region with the patient's blood, being made with whole blood, washed erythrocytes, plasma and $0.9 \%$ saline. After three hours, there was a reaction in the injections made with whole blood and mainly with erythrocytes, with the appearance of local ecchymosis and hardened, edematous and hyperemic lesions in the region. No reactions were observed in the injections made with saline and plasma. Since then, she was treated with fluoxetine, clonazepam and sent to psychiatric care, showing control of injuries.

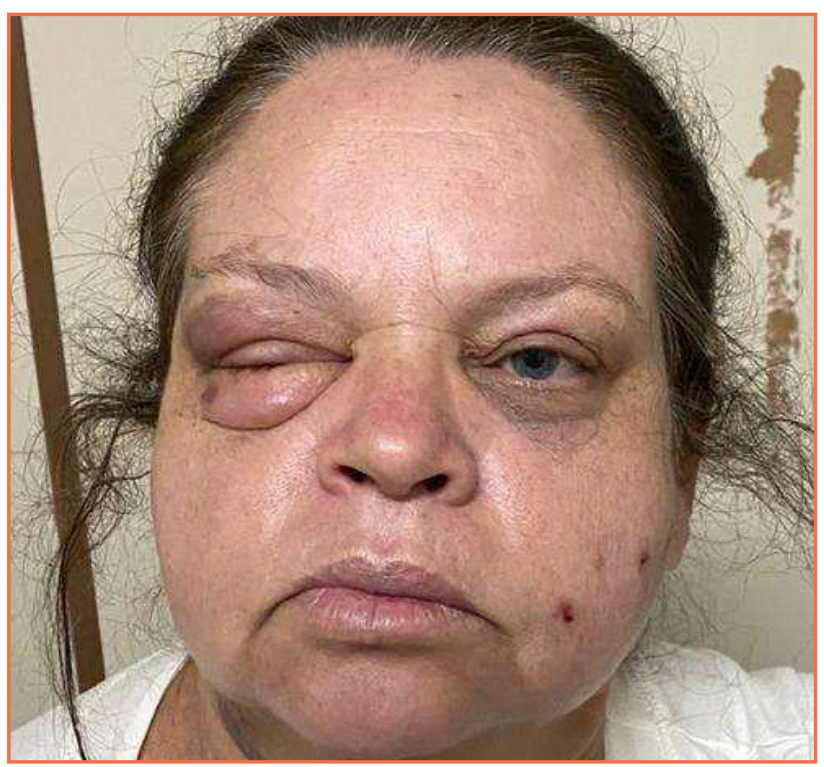

Figure 1. Eyelid ecchymosis and bilateral edema

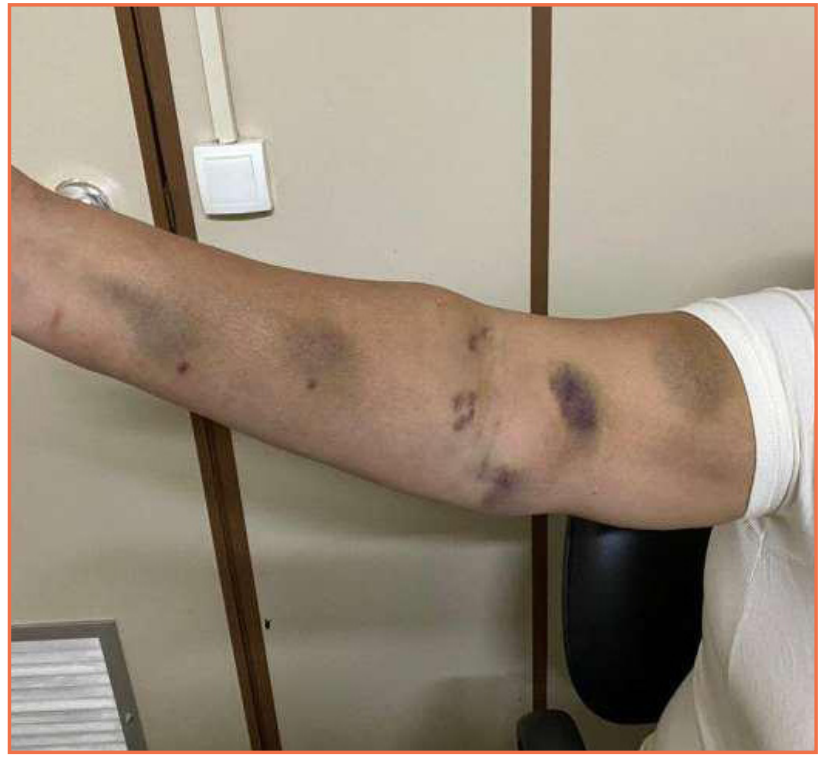

Figure 2. Nodular and painful cutaneous lesions

\section{CONCLUSION}

The purpose of the case report is to bring a rare disease with little description in the literature and difficult diagnosis. Despite its rarity, GDS should be a part of differential diagnosis of hematological findings, especially in women with depression/anxiety. 